\title{
Genetic Variants in the Hedgehog Interacting Protein Gene Are Associated with the FEV1/FVC Ratio in Southern Han Chinese Subjects with Chronic Obstructive Pulmonary Disease
}

\author{
Zili Zhang, ${ }^{1}$ Jian Wang, ${ }^{1,2}$ Zeguang Zheng, ${ }^{1}$ Xindong Chen, ${ }^{3}$ Xiansheng Zeng, \\ Yi Zhang, ${ }^{5}$ Defu Li, ${ }^{1}$ Jiaze Shu, ${ }^{1}$ Kai Yang, ${ }^{1}$ Ning Lai, ${ }^{1}$ Lian Dong, ${ }^{1}$ and Wenju Lu ${ }^{1,2,6}$ \\ ${ }^{1}$ State Key Laboratory of Respiratory Diseases, Guangzhou Institute of Respiratory Disease, The First Affiliated Hospital, \\ Guangzhou Medical University, Guangzhou, Guangdong, China \\ ${ }^{2}$ Department of Pulmonary and Critical Care Medicine, The Johns Hopkins University School of Medicine, Baltimore, MD, USA \\ ${ }^{3}$ Department of Respiratory Medicine, Lufeng People's Hospital, Lufeng, Guangdong, China \\ ${ }^{4}$ Department of Respiratory Medicine, Xiangyang Central Hospital, Xiangyang, Hubei, China \\ ${ }^{5}$ Department of Respiratory Medicine, The Second Affiliated Hospital, Guangzhou Medical University, Guangzhou, Guangdong, China \\ ${ }^{6}$ Department of Laboratory Medicine, The First Affiliated Hospital, Guangzhou Medical University, Guangzhou, Guangdong, China
}

Correspondence should be addressed to Wenju Lu; wlu92@yahoo.com

Received 15 May 2017; Revised 11 July 2017; Accepted 18 July 2017; Published 27 August 2017

Academic Editor: Salvatore Battaglia

Copyright ( $\odot 2017$ Zili Zhang et al. This is an open access article distributed under the Creative Commons Attribution License, which permits unrestricted use, distribution, and reproduction in any medium, provided the original work is properly cited.

Background. Convincing evidences have demonstrated the associations between HHIP and FAM13a polymorphisms and COPD in non-Asian populations. Here genetic variants in HHIP and FAM13a were investigated in Southern Han Chinese COPD. Methods. A case-control study was conducted, including 989 cases and 999 controls. The associations between SNPs genotypes and COPD were performed by a logistic regression model; for SNPs and COPD-related phenotypes such as lung function, COPD severity, pack-year of smoking, and smoking status, a linear regression model was employed. Effects of risk alleles, genotypes, and haplotypes of the 3 significant SNPs in the HHIP gene on $\mathrm{FEV}_{1} / \mathrm{FVC}$ were also assessed in a linear regression model in COPD. Results. The mean $\mathrm{FEV}_{1} / \mathrm{FVC} \%$ value was 46.8 in combined COPD population. None of the 8 selected SNPs apparently related to COPD susceptibility. However, three SNPs (rs12509311, rs13118928, and rs182859) in HHIP were associated significantly with the $\mathrm{FEV}_{1} / \mathrm{FVC} \%\left(P_{\max }=4.1\right.$ $\times 10^{-4}$ ) in COPD adjusting for gender, age, and smoking pack-years. Moreover, statistical significance between risk alleles and the $\mathrm{FEV}_{1} / \mathrm{FVC} \%\left(P=2.3 \times 10^{-4}\right)$, risk genotypes, and the $\mathrm{FEV}_{1} / \mathrm{FVC} \%\left(P=3.5 \times 10^{-4}\right)$ was also observed in COPD. Conclusions. Genetic variants in $\mathrm{HHIP}$ were related with $\mathrm{FEV}_{1} / \mathrm{FVC}$ in COPD. Significant relationships between risk alleles and risk genotypes and $\mathrm{FEV}_{1} / \mathrm{FVC}$ in COPD were also identified.

\section{Introduction}

Chronic Obstructive Pulmonary Disease (COPD) is a complex disease characterized by airflow limitation that is not completely reversible. It is projected to rise to rank fifth in disease burden by 2020 around the world [1], and in USA, it ranks as the third leading cause of death [2]. Although tobacco smoking was suggested to be the major adverse factor for the progress of COPD, about $85 \%$ of the smokers did not develop into clinically relevant airflow obstruction [3], implying that genetic susceptibility might play a crucial role in the development of COPD. However, these genetic factors are not yet fully understood. Few susceptibility genes other than $\alpha_{1}$-ANTITRYPSIN have been convincingly identified yet [4].

Genome-wide association studies (GWAS), which have revolutionized the identification of susceptibility genes for polygenic diseases, figured out statistically significant relations regarding the FAM13a and HHIP and COPD in nonAsian populations [5-8]. Evidences identified that FAM13a and HHIP might be involved in the etiology of COPD [9, 10]. For instance, several GWAS studies have showed the 
significant associations between the HHIP loci and COPD susceptibility $[9,11]$. In view of the great differences in genetic profiles of different ethnicity, replication works in other populations and with more SNPs are warranted. Therefore, the genetic relationships between the FAM13a and HHIP genes and COPD were conducted by the Southern Han Chinese COPD case-control study. We wanted to explore whether a selection of SNPs in FAM13a and HHIP was related to COPD and COPD-related phenotypes.

\section{Methods and Materials}

2.1. Study Population. The study design and subject recruitment have been described previously [12]. In short, all participants were genetically unrelated ethnic Southern Han Chinese and from Guangdong and Hubei Province, 40 to 80 years old. All 989 hospitalized COPD patients manifested after bronchodilator the $\mathrm{FEV}_{1} / \mathrm{FVC} \%$ values of $<0.7$ and were successively recruited from September 2010 to September 2013 in the Department of Respiratory Medicine (an ongoing project). "Study I" involved 594 cases and 600 controls from the First Affiliated Hospital of Guangzhou Medical University (Guangzhou, Guangdong, China). "Study II" was conducted on participants (395 cases and 399 controls) derived from Xiangyang, Central Hospital (Xiangyang, Hubei, China), to verify the results from the Study I population. The 999 control subjects demonstrated normal lung function (postbronchodilator $\mathrm{FEV}_{1}>80 \%$ predicted; the $\mathrm{FEV}_{1} / \mathrm{FVC} \%>0.7$ ) and were randomly recruited from the Health Examination Center of the same hospital during the same time period when patients were recruited to maximally match the situation of the regarding cases; efforts were made to frequencymatch COPD by age ( \pm 5 years) and sex. Participants were excluded with regard to concomitant respiratory disorders, lung surgery, and pregnancy, and so forth before enrollment.

2.2. Data Collection. Prebronchodilator and postbronchodilator spirometry were conducted on subjects according to standardized protocol with the EasyOne Spirometer (NDD, Inc., Andover, MA) [13]. Post-bronchodilator spirometry was measured probably 20 minutes after performing $180 \mu \mathrm{g}$ of albuterol via metered dose inhaler. Few patients could not finish at least three complete spirometries or their values of measurements mildly exceeded spirometric criteria; the data of those patients included were determined by the investigators' discretion. Predicted FEV1\% and FEV1/FVC\% were evaluated using the Global Lung Function Initiative (GLI) equations [14]. Airway obstruction was diagnosed by the GLI definition of FEV1/FVC < the Lower Limit of Normal (LLN) and $z$-score. Data was described by the mean \pm SD (strictly $1.96 z$-scores), which extended from the 2.5 th to the 97.5th centile of the distribution ( $z$-scores indicate how many standard deviations a measurement is from its predicted value). Categorization of the severity of airway obstruction was made using the four-category scale (mild: $z \geq-2$; moderate: $-2>z \geq-2.5$; moderately severe: $-2.5>z \geq$ -3 ; severe: $-3>z \geq-4,-4>z$ ) [15]. Prior to formal recruitment, informed consent was signed by each of the qualified subjects as written form, and structured questionnaires were conducted by professional staff to collect information on demographic data and environmental exposure history such as tobacco cigarette smoke and so forth. Subjects were subgrouped as smokers, ex-smokers, and nonsmokers. The definitions of nonsmokers, ex-smokers, nonsmokers, and packyears of smoking were detailed in our earlier study [16]. After the interview, a $\sim 5 \mathrm{ml}$ venous blood sample was collected from each participant. This study was approved by each participating center's Institutional Ethical Committee and was administrated by the principles of the Declaration of Helsinki (Ethics Committee of the First Affiliated Hospital: GZMC 2010-03-28; Xiangyang Central Hospital of Hubei: 2011-01-23).

2.3. Polymorphisms Selection and Genotyping Assays. Based on the findings from the GWAS of COPD in non-Asian populations, three top SNPs (rs1903003, rs2869967, and rs7671167) in FAM13a gene and five top SNPs (rs12504628, rs12509311, rs13118928, rs1512281, and rs1828591) in HHIP gene, which were found to be significantly associated with COPD risk, were selected [5-8] (the top SNP defined as $P$ value was the most significant). Because we chose these SNPs based on the reported top findings of COPD GWAS in non-Asian populations, rather than the database such as dbSNP and Hapmap, we just did the replication analyses in the present study without considering the linkage disequilibrium $(L D)\left(D^{\prime} \geq\right.$ $0.9864, r^{2} \geq 0.9621$ between the five SNPs in HHIP in controls) (see Table S1 in Supplementary Material available online at https://doi.org/10.1155/2017/2756726).

QIAGEN Blood DNA Kit was employed to extract the genomic DNA of each participant. ABI PRISM 7500 Sequence Detection System (Applied Biosystems, Foster City, CA) with allelic discrimination method was chosen for genotyping [17]. Primers and probes assay as well as polymerase chain reaction has been described sufficiently in our earlier study [16]. The genotypes were automatically calculated by Sequence Detection Systems software 2.3 (Applied BioSystems). The accordance rate of each SNP was $100 \%$ for the duplicates of $10 \%$ of samples.

2.4. Statistical Analysis. Baseline characteristics were analyzed for quantitative traits using $t$-tests and for binary traits using a two-sided $\chi^{2}$ test. Goodness of fit to the HardyWeinberg equilibrium expectation in controls was also assessed by the $\chi^{2}$ for each SNP. The associations between COPD susceptibility and SNP genotypes were calculated by an unconditional logistic regression model after adjustment for gender, age, and smoking pack-years under different genetic models. The associations between COPD-related phenotypes and the three significant SNPs were tested by a linear regression model after adjusting for gender, age, and smoking pack-years with an additive genetic model. Effects of risk alleles and genotypes of the 3 significant SNPs on the FEV1/FVC\% were also calculated using a linear regression model adjusting for gender, age, and smoking pack-years. To control the family-wise type I error rate at a 0.05 level, a Bonferroni correction was applied. With 8 SNPs between comparisons, each individual 2-sided test was considered 
statistically significant relative to a 0.006 significance level. Spirometry predicted values and $z$-scores were derived for each subject in each dataset using prediction equations from GLI-2012 [14] using specially developed GLI-ExcelCalculator in the supplementary file from the Quanjer et al. study [15]. Statistical analyses were all evaluated using the SAS9.2 software and statistical power measured by Quanto 1.2 .

\section{Results}

3.1. Characteristics of the Study Population. The characteristics of the combined 989 COPD patients and 999 controls were described in Table 1. Briefly, the COPD cases and controls appeared to be age matched $(P=0.807)$. As expected, COPD cases indicated a more serious condition on pulmonary function compared to controls. The pack-year of cigarette smoking and the frequency of male in COPD were greater than that in control subjects. The ex-smokers were more likely suffering from COPD, while more nonsmokers or smokers were to be control subjects. Among the 989 COPD cases, 155 (15.7\%) were defined as mild COPD, 156 (15.8\%) as moderate, $368(37.2 \%)$ as severe, and $310(31.3 \%)$ as very severe. The spearman coefficients between the FEV1/FVC\% and FEV1 were 0.5526 and -0.0718 in subjects with COPD and controls, respectively (Table S2). Moreover, Study I and Study II revealed almost identical change tendency as the combined population (principal components analysis for heterogeneity test between the two groups, $P=0.226$, Table S3).

3.2. Genetic Association. Case-control analyses exploring further associations identified that none of the SNPs was significantly related to COPD risks without adjustment. Linear regression analyses showed that three SNPs (rs12509311, rs13118928, and rs1828591) in the HHIP gene were significantly associated with the FEV1/FVC\% in COPD with adjustment in combined population $\left(P_{\max }=4.1 \times 10^{-4}\right)$ (Table 2$)$. Therefore, additional models with adjusting for gender, age, and pack-years of smoking were conducted for further analyses. Similarly, none of the SNPs were figured out to be genetic associations with COPD susceptibility (Table 3). However, rs12509311, rs13118928, and rs1828591 were only significantly associated with the FEV1/FVC\% in COPD $\left(P=4.1 \times 10^{-4}, 2.8\right.$ $\times 10^{-4}$, and $4.1 \times 10^{-4}$, resp.) (Table 4$)$ rather than in the controls (Table S4). Risk alleles and genotypes of the three SNPs analyses revealed that there were significant associations between risk alleles and the FEV1/FVC $\%\left(P=2.3 \times 10^{-4}\right)$ as well as between risk genotypes and the FEV1/FVC\% $(P=3.5$ $\times 10^{-4}$ ) in subjects with COPD after adjusting for gender, age, and pack-years of smoking (Table 5).

\section{Discussion}

To date, COPD remains a major worldwide and increasing health problem incurred by multiple genetic and environmental factors [9]. GWAS have identified several susceptibility genes for COPD, including FAM13a, HHIP, CHRNA3/ CHRNA5/IREB2, RIN3, MMP3/MMP12, and TGFB2 [7, 8, 10, $11,18-23]$. These loci identified in GWAS were conducted not only in population of European and African descent, but also in Asians [24-26]. For FAM13a, the susceptibility for COPD was also found in Hispanics [27]. Recently, studies have revealed the associations between HHIP and lung function $[24,28]$. Kim et al. found that, in the KOLD cohort study, two SNPs (rs11938704 and rs10013495) near HHIP were significantly associated with FEV1 $(P=0.0001$ and 0.001 , resp.) in COPD [28]. However, evidences from those studies have not yet been reached for the other COPD phenotypes. In the current case-control study, we explored the role of multiple variants of FAM13a and HHIP and assessed their relationships not only with COPD, but also with COPDrelated phenotypes in above described Southern Chinese Han population. In this study, we analyzed 3 top SNPs in FAM13a gene and 5 top SNPs in HHIP gene, which previously showed statistical significance based on GWAS, but the genetic associations with COPD susceptibility were not identified in the current study. Genetic variants in HHIP were associated with the FEV1/FVC\% in COPD cases with adjustment for gender, age, and pack-years of smoking. In addition, we also identified significant relationships between risk alleles or risk genotypes and the FEV1/FVC\% in COPD. These results indicated that the HHIP gene might contribute to the variation of the FEV1/FVC\%.

It is biologically possible that HHIP may be involved in the etiology of COPD. The gene of HHIP encodes a membrane glycoprotein, which is an endogenous antagonist for the protein of Sonic Hedgehog ( $\mathrm{SHH})[29,30]$. The evolutionarily highly conserved hedgehog signaling pathway is functionally implicated in a variety of physiological or pathological processes, including lung organogenesis, embryogenesis, chronic inflammation, and carcinogenesis, as well as for response of the airway epithelium exposed to smoking [31-33]. Hedgehog signaling by HHIP is playing crucial roles in lung morphogenesis, especially in the stage during early lung branching. Knockout of HHIP in mice led to the inhibition of lung bud branching and neonatal respiratory failure. Changes of HHIP protein or its expression in humans might change the development of lung or repair mechanisms [34, 35]. Earlier GWAS studies demonstrated significant associations between the HHIP loci and COPD risk $[18,21]$. The associations of $H H I P$ with COPD risk were also shown in the Rotterdam study and the Polish cohort and one study among Southwestern Chinese Han population [21, 36]. However, the association between HHIP and COPD risk was not identified in the Southern Chinese Han population in this study. The explanations for these conflicting results might be as follows: first, ethnic differences contributed to this variability $[37,38]$. For instance, study identified novel association between certain genes with chronic diseases in Hispanic population, but in non-Hispanic the significance was rather limited, including the two novel loci (rs858249 and rs286499) in or near the genes KLHL7/NUPL2 and DLG2 [27]. Similarly, associations identified in European-ancestry population might be less significant in non-European populations due to difference in ethnicity [39]. Second, the reason might be due to the different consumption of cigarettes. In the previously mentioned Rotterdam study, SNPs in HHIP were more associated with COPD in the subgroup of heavy 


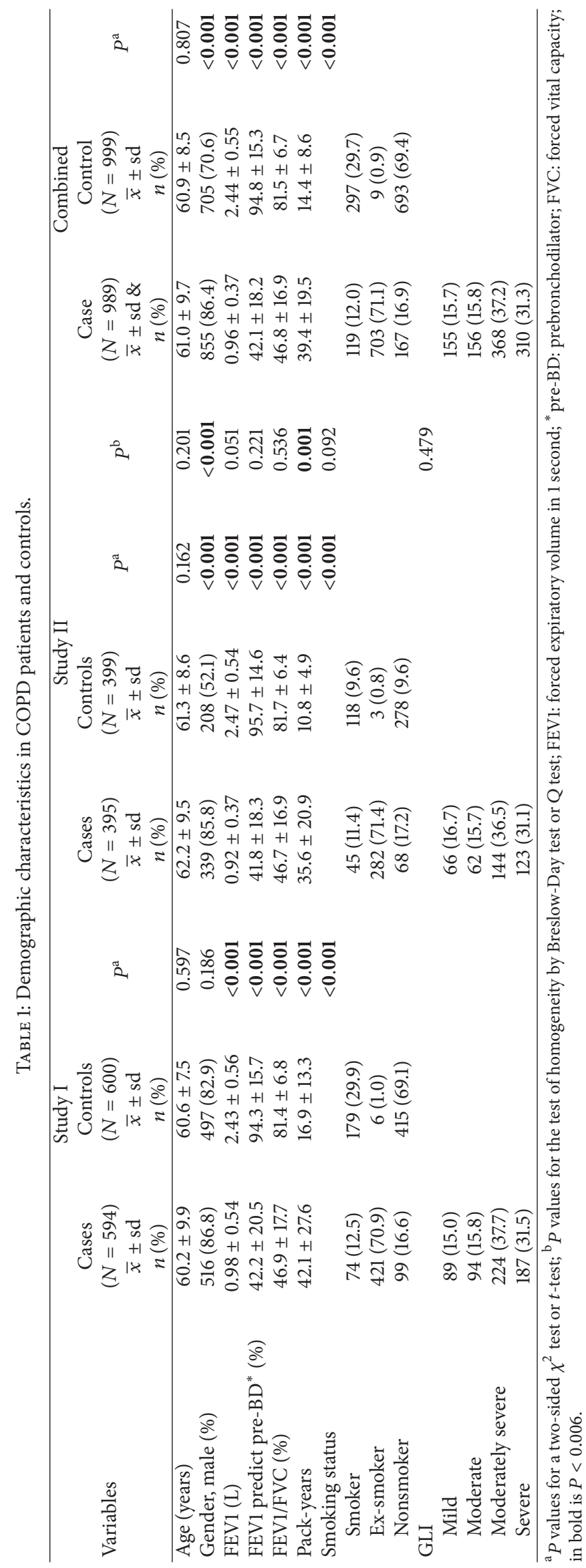




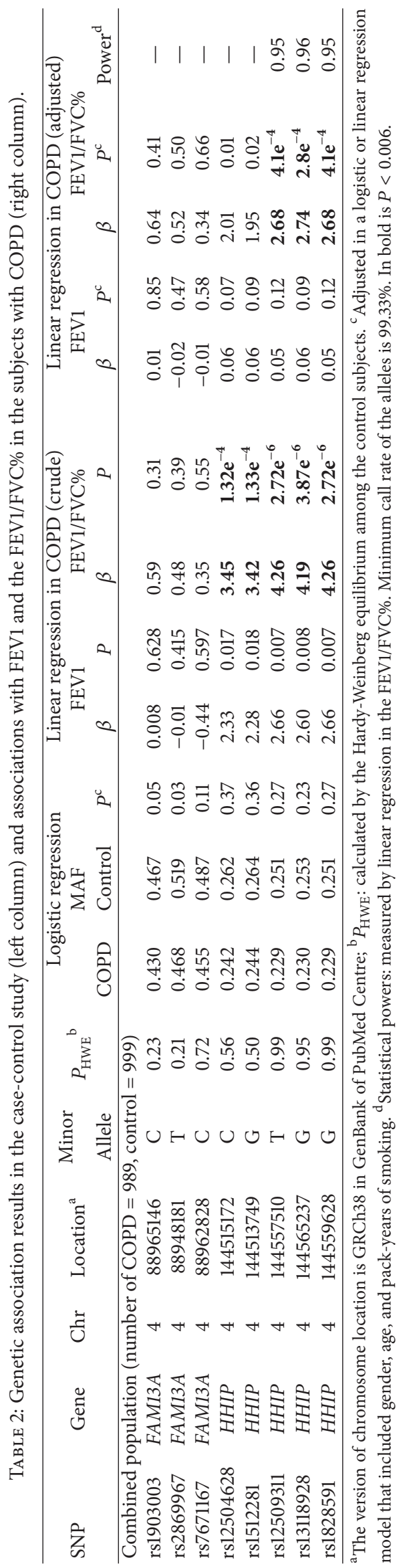


TABLE 3: Analyses of associations between 3 SNPs in the HHIP gene and COPD.

\begin{tabular}{|c|c|c|c|c|c|}
\hline \multirow{2}{*}{ Genotypes/alleles } & \multirow{2}{*}{$\begin{array}{l}\text { Case } \\
n(\%)\end{array}$} & \multirow{2}{*}{$\begin{array}{c}\text { Control } \\
n(\%)\end{array}$} & \multirow{2}{*}{$\begin{array}{c}\text { Crude } \\
\text { OR }(95 \% \mathrm{CI})\end{array}$} & \multicolumn{2}{|c|}{ Adjusted } \\
\hline & & & & OR (95\% CI) & $P^{\mathrm{a}}$ \\
\hline Total number of subjects & 989 & 999 & & & \\
\hline Total number of alleles & 1978 & 1998 & & & \\
\hline \multicolumn{6}{|l|}{$r s 12509311 C>T$} \\
\hline \multicolumn{6}{|l|}{ Codominant model } \\
\hline $\mathrm{CC}$ & $581(59.0)$ & $559(56.1)$ & 1.00 (ref.) & 1.00 (ref.) & \\
\hline $\mathrm{CT}$ & $356(36.1)$ & $374(37.6)$ & $0.92(0.76-1.10)$ & $0.85(0.62-1.17)$ & 0.32 \\
\hline $\mathrm{TT}$ & $48(4.9)$ & $63(6.3)$ & $0.73(0.50-1.09)$ & $0.69(0.38-1.27)$ & 0.24 \\
\hline Additive model & & & $0.89(0.77-1.03)$ & $0.84(0.66-1.07)$ & 0.16 \\
\hline \multicolumn{6}{|l|}{ Dominant model } \\
\hline $\mathrm{CT}+\mathrm{TT}$ & $404(41.0)$ & $437(43.9)$ & $0.89(0.74-1.06)$ & $0.82(0.61-1.11)$ & 0.21 \\
\hline \multicolumn{6}{|l|}{ Recessive model } \\
\hline $\mathrm{CC}+\mathrm{CT}$ & $937(95.1)$ & $933(93.7)$ & 1.00 (ref.) & 1.00 (ref.) & \\
\hline TT & $48(4.9)$ & $63(6.3)$ & $0.76(0.52-1.12)$ & $0.74(0.41-1.33)$ & 0.31 \\
\hline \multicolumn{6}{|l|}{$r s 13118928 A>G$} \\
\hline \multicolumn{6}{|l|}{ Codominant model } \\
\hline $\mathrm{AA}$ & $581(58.9)$ & $559(56.0)$ & 1.00 (ref.) & 1.00 (ref.) & \\
\hline GA & $358(36.3)$ & $374(37.4)$ & $0.92(0.77-1.11)$ & $0.85(0.62-1.17)$ & 0.33 \\
\hline GG & $48(4.8)$ & $66(6.6)$ & $0.70(0.47-1.03)$ & $0.69(0.38-1.25)$ & 0.22 \\
\hline Additive model & & & $0.88(0.76-1.02)$ & $0.84(0.66-1.07)$ & 0.15 \\
\hline \multicolumn{6}{|l|}{ Dominant model } \\
\hline $\mathrm{GA}+\mathrm{GG}$ & $406(41.1)$ & $440(44.0)$ & $0.89(0.74-1.06)$ & $0.82(0.61-1.11)$ & 0.20 \\
\hline \multicolumn{6}{|l|}{ Recessive model } \\
\hline $\mathrm{AA}+\mathrm{GA}$ & $939(95.2)$ & $933(93.4)$ & 1.00 (ref.) & 1.00 (ref.) & \\
\hline GG & $48(4.8)$ & $66(6.6)$ & $0.72(0.49-1.06)$ & $0.73(0.40-1.31)$ & 0.29 \\
\hline \multicolumn{6}{|l|}{$r s 1828591 A>G$} \\
\hline \multicolumn{6}{|l|}{ Codominant model } \\
\hline $\mathrm{AA}$ & $581(59.0)$ & $559(56.1)$ & 1.00 (ref.) & 1.00 (ref.) & \\
\hline GA & $356(36.1)$ & $374(37.6)$ & $0.92(0.76-1.10)$ & $0.85(0.62-1.17)$ & 0.32 \\
\hline GG & $48(4.9)$ & $63(6.3)$ & $0.73(0.50-1.09)$ & $0.69(0.38-1.27)$ & 0.24 \\
\hline Additive model & & & $0.89(0.77-1.03)$ & $0.84(0.66-1.07)$ & 0.16 \\
\hline \multicolumn{6}{|l|}{ Dominant model } \\
\hline $\mathrm{GA}+\mathrm{GG}$ & $404(41.0)$ & $437(43.9)$ & $0.89(0.74-1.06)$ & $0.82(0.61-1.11)$ & 0.21 \\
\hline \multicolumn{6}{|l|}{ Recessive model } \\
\hline $\mathrm{AA}+\mathrm{GA}$ & $937(95.1)$ & $933(93.7)$ & 1.00 (ref.) & 1.00 (ref.) & \\
\hline GG & $48(4.9)$ & $63(6.3)$ & $0.76(0.52-1.12)$ & $0.74(0.41-1.33)$ & 0.31 \\
\hline
\end{tabular}

${ }^{a}$ Adjusted in a logistic regression model that included gender, age, and pack-years of smoking.

smoker, and genetic variation near the HHIP gene was significantly associated with risk of COPD depending on the quantity of pack-years of smoking. However, the relatively mild smoking levels of the population in our study might result in the nonsignificant association between SNPs in HHIP and COPD susceptibility (Table S5). Third, the reasons could be due to the differences in COPD severity. In the Polish cohort, SNPs in the $4 \mathrm{q} 31$ chromosome region were greatly associated with severe COPD [36] and the HHIP SNP rs10519717 was associated with the severity of COPD in the Southwestern Chinese Han population. Fourth, the reasons could be differences determined by characteristics of population such as age, gender distribution, and height. For instance, earlier GWAS found out that genetic variants in
HHIP were significantly related to adult height in European and Korean populations [21, 40]. Fifth, differences resulted from measurements of lung function. In most of the constituent studies, postbronchodilator spirometry was not measured. However, previous study reported that substantial misclassification would occur by using prebronchodilator spirometry to diagnose COPD in mild COPD case subjects [41]. In the present study, postbronchodilator spirometry was measured and mild COPD cases were included. Finally, the discrepancies might come from different pathologic stages of airflow obstruction. Postbronchodilator spirometry was applied for the detection of airflow obstruction, which was regarded as the formal diagnosis of COPD. Patients with partly or fully reversible airflow obstruction might have 


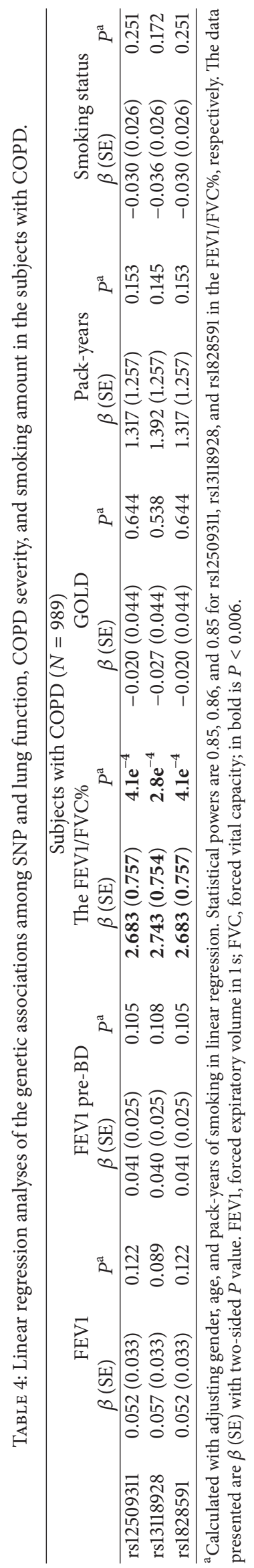


TABLE 5: Effect of risk allele and genotypes of 3 SNPs in the HHIP gene on the FEV1/FVC\% in the subjects with COPD.

\begin{tabular}{lcccc}
\hline & \multicolumn{3}{c}{ Subjects with COPD $(N=989)$} \\
& Crude & Adjusted \\
& $\beta(\mathrm{SE})$ & $P$ & $\beta(\mathrm{SE})$ & $P^{\mathrm{a}}$ \\
\hline Risk allele $^{\mathrm{b}}$ & $1.328(0.309)$ & $\mathbf{1 . 9 \mathrm { e } ^ { - \mathbf { 5 } }}$ & $1.077(0.292)$ & $\mathbf{2 . 3 \mathrm { e } ^ { - \mathbf { 4 } }}$ \\
Risk genotypes $^{\mathrm{b}}$ & $2.058(0.490)$ & $\mathbf{2 . 7 \mathrm { e } ^ { - \mathbf { 5 } }}$ & $1.651(0.462)$ & $\mathbf{3 . 5 \mathrm { e } ^ { - \mathbf { 4 } }}$ \\
\hline
\end{tabular}

${ }^{a}$ Adjusted in a linear regression model that included gender, age, and packyears of smoking; risk allele means number of the three SNPs in combination.

${ }^{\mathrm{b}}$ Genotype combinations in HHIP: rs12509311T variant genotypes (CT or TT), rs13118928G variant genotypes (AG or GG), or rs1828591G variant genotypes (AG or GG) are defined as risk genotypes. Risk genotype means number of variant genotypes in combination. The data presented are $\beta$ (SE) with two-sided $P$ value. In bold is $P<0.006$.

fundamentally different pathological development contributing to airflow obstruction. The underlying bias was that there might be some patients with misclassification of either an asthma diagnosis or the COPD diagnosis. Both asthma and COPD are common diagnoses and could coexist in the same cases. If the SNP effects were specific to asthma and not present in COPD, the contribution of the SNPs to COPD would be overestimated if including the patients with asthma. However, we investigated the effects with exclusion of patients with known asthma from the case subjects in a subset of the data with asthma diagnosis available.

Furthermore, many previous studies have demonstrated significant genetic relationships between HHIP loci and the FEV1/FVC\% such as in the GWAS of the Framingham Heart Study population [7], the Cohorts for Heart and Aging Research in Genomic Epidemiology and SpiroMeta consortia [5, 42], Evaluation of COPD Longitudinally to Identify Predictive Surrogate Endpoints (ECLIPSE), and International COPD Genetics Network (ICGN) subjects [43]. The FEV1/FVC\% is an important quantitative characteristic of COPD. The decreased FEV1/FVC\%, an indicator of airflow obstruction that is independent of lung size, is the important criterion for defining an obstructive ventilator defect [44]. We also identified a significant association between SNPs in HHIP and the FEV1/FVC\% in COPD in Southern Chinese Han population. The results showed that rs12509311, rs13118928, and rs1828591 were associated with the FEV1/FVC\% among cases only. While some other studies did not draw such a conclusion [24, 28], potential explanations might be the differences in genetic heterogeneity, statistical power, and population characteristic. Generally, genetic heterogeneity would be an important factor to explain this conflict. Even if the same genetic variant is involved in each population, the $L D$ relationships of this variant with neighboring genetic polymorphisms might also vary between ethnic groups. Moreover, our statistical powers were all $\geq 95 \%$ to support our claim. The FEV1/FVC\% is also determined by population characteristics including height and heritability. Given that COPD airflow obstruction is typically determined by the FEV1/FVC\%, the current study suggested that HHIP was associated with COPD airflow obstruction. Besides, the relationship between SNPs in HHIP and the other COPD phenotypes were also observed, but significant associations were not demonstrated, including $\mathrm{FEV}_{1}$, COPD severity, and smoking status. Except the above-mentioned three explanations (genetic heterogeneity, statistical power, and population characteristic), differences in smoking exposure, current smoking status, entry criteria, and geographic origin of the population might also make a contribution to phenotypic heterogeneity and lead to the discrepancies. Additionally, significant relationships between risk alleles or risk genotypes and the FEV1/FVC\% in COPD also were demonstrated in our study. Explanations on associations between SNPs in HHIP and the FEV1/FVC\% could be also applicable here because of risk alleles or risk genotypes actually resulting from the combination of number of the three risk SNPs. For further study, functional experiments should be needed.

In conclusion, genetic variants in $H H I P$ were found to be associated with the FEV1/FVC\% in COPD cases. However, the relationship between HHIP and FAM13a polymorphisms and COPD susceptibility was not identified. Significant relationships between risk alleles and the $\mathrm{FEV1/FVC \%} \mathrm{as} \mathrm{well} \mathrm{as}$ risk genotypes and the FEV1/FVC\% in subjects with COPD were identified. Given the uniform conclusions in Chinese populations, replication studies with more populations and more SNPs are required in the future study.

\section{Abbreviations \\ COPD: Chronic obstructive pulmonary disease \\ $\mathrm{FEV}_{1}$ : Forced expiratory volume in $1 \mathrm{~s}$ \\ FVC: $\quad$ Forced vital capacity \\ FAM13a: Family with sequence similarity 13 , member a \\ HHIP: Hedgehog interacting protein \\ GWAS: Genome-wide association study \\ SNP: Single-nucleotide polymorphism.}

\section{Disclosure}

The funding sponsors had no role in the design of the study; in the collection, analyses, or interpretation of data; in the writing of the manuscript; and in the decision to publish the results.

\section{Conflicts of Interest}

The authors declare that they have no conflicts of interest.

\section{Authors' Contributions}

Wenju Lu conceived and designed the experiments. Zili Zhang, Defu Li, Jiaze Shu, Kai Yang, Ning Lai, and Lian Dong performed the experiments; Zili Zhang analyzed the data; Zeguang Zheng, Xindong Chen, Xiansheng Zeng, and Yi Zhang contributed reagents/materials/analysis tools; Zili Zhang wrote the paper; Wenju Lu and Jian Wang reviewed and revised the manuscript. Zili Zhang, Jian Wang, and Zeguang Zheng contributed equally to this article. 


\section{Acknowledgments}

This work was supported by grants from National Natural Science Foundation of China (81520108001 and 81700043), the 973 Key Scheme of China (2015CB553406), Guangdong Province Universities and Colleges Pearl River Scholar Funded Scheme (2014, W. Lu), Guangzhou Department of Education for Innovative Team (13C08), and Guangdong Natural Science Foundation (2016A030313593).

\section{References}

[1] K. F. Rabe, S. Hurd, A. Anzueto et al., "Global strategy for the diagnosis, management, and prevention of chronic obstructive pulmonary disease: GOLD executive summary," The American Journal of Respiratory and Critical Care Medicine, vol. 176, no. 6, pp. 532-555, 2007.

[2] A. M. Minino, J. Xu, and K. D. Kochanek, National Vital Statistics Reports: Deaths: Preliminary Data for 2008, vol. 59, National Center for Health Statistics, Hyattsville, Md, USA, 2010.

[3] C. M. Fletcher, "Natural history of chronic bronchitis," British Medical Journal, vol. 1, no. 6025, pp. 1592-1593, 1976.

[4] P. O. Ganrot, C.-B. Laurell, and S. Eriksson, "Obstructive lung disease and trypsin inhibitors in $\alpha 1$-antitrypsin deficiency," Scandinavian Journal of Clinical and Laboratory Investigation, vol. 19, no. 3, pp. 205-208, 1967.

[5] E. Repapi, I. Sayers, L. V. Wain et al., "Genome-wide association study identifies five loci associated with lung function," Nature Genetics, vol. 42, no. 1, pp. 36-44, 2010.

[6] M. G. Foreman, M. Campos, and J. C. Celedón, "Genes and chronic obstructive pulmonary disease," Medical Clinics of North America, vol. 96, no. 4, pp. 699-711, 2012.

[7] J. B. Wilk, T.-H. Chen, D. J. Gottlieb et al., "A genomewide association study of pulmonary function measures in the framingham heart study," PLoS Genetics, vol. 5, no. 3, Article ID e1000429, 2009.

[8] M. H. Cho, N. Boutaoui, B. J. Klanderman et al., "Variants in FAM13A are associated with chronic obstructive pulmonary disease," Nature Genetics, vol. 42, no. 3, pp. 200-202, 2010.

[9] D. M. Mannino and A. S. Buist, "Global burden of COPD: risk factors, prevalence, and future trends," The Lancet, vol. 370, no. 9589, pp. 765-773, 2007.

[10] M. H. Cho, M.-L. N. McDonald, X. Zhou et al., "Risk loci for chronic obstructive pulmonary disease: A genome-wide association study and meta-analysis," The Lancet Respiratory Medicine, vol. 2, no. 3, pp. 214-225, 2014.

[11] M. Hardin, J. Zielinski, E. S. Wan et al., "CHRNA3/5, IREB2, and ADCY2 are associated with severe chronic obstructive pulmonary disease in Poland," American Journal of Respiratory Cell and Molecular Biology, vol. 47, no. 2, pp. 203-208, 2012.

[12] W. Lu, Z. Zheng, X. Chen et al., "Study design and interim outcomes of Guangzhou Institute of respiratory disease COPD Biobank," COPD: Journal of Chronic Obstructive Pulmonary Disease, vol. 13, no. 2, pp. 203-213, 2016.

[13] M. R. Miller, J. Hankinson, V. Brusasco et al., "Standardisation of spirometry," European Respiratory Journal, vol. 26, no. 2, pp. 319-338, 2005.

[14] P. H. Quanjer, S. Stanojevic, T. J. Cole et al., "Multi-ethnic reference values for spirometry for the 3-95-yr age range: the global lung function 2012 equations," European Respiratory Journal, vol. 40, no. 6, pp. 1324-1343, 2012.

[15] P. H. Quanjer, J. J. Pretto, D. J. Brazzale, and P. W. Boros, "Grading the severity of airways obstruction: New wine in new bottles," European Respiratory Journal, vol. 43, no. 2, pp. 505512, 2014.

[16] Z. Zhang, J. Wang, J. He et al., "Genetic Variants in MUC4 Gene Are Associated with Lung Cancer Risk in a Chinese Population," PLoS ONE, vol. 8, no. 10, Article ID e77723, 2013.

[17] K. J. Livak, "Allelic discrimination using fluorogenic probes and the $5^{\prime}$ nuclease assay," Genetic Analysis: Biomolecular Engineering, vol. 14, no. 5-6, pp. 143-149, 1999.

[18] S. G. Pillai, D. Ge, G. Zhu et al., "A genome-wide association study in chronic obstructive pulmonary disease (COPD): identification of two major susceptibility loci," PLoS Genetics, vol. 5, no. 3, Article ID e1000421, 2009.

[19] D. L. DeMeo, T. Mariani, S. Bhattacharya et al., "Integration of Genomic and Genetic Approaches Implicates IREB2 as a COPD Susceptibility Gene," American Journal of Human Genetics, vol. 85, no. 4, pp. 493-502, 2009.

[20] R. P. Young, R. J. Hopkins, B. A. Hay, M. J. Epton, P. N. Black, and G. D. Gamble, "Lung cancer gene associated with COPD: Triple whammy or possible confounding effect?" European Respiratory Journal, vol. 32, no. 5, pp. 1158-1164, 2008.

[21] Y. M. T. A. Van Durme, M. Eijgelsheim, G. F. Joos et al., "Hedgehog-interacting protein is a COPD susceptibility gene: The Rotterdam study," European Respiratory Journal, vol. 36, no. 1, pp. 89-95, 2010.

[22] R. P. Young, C. F. Whittington, R. J. Hopkins et al., "Chromosome 4q31 locus in COPD is also associated with lung cancer," European Respiratory Journal, vol. 36, no. 6, pp. 1375-1382, 2010.

[23] R. P. Young, R. J. Hopkins, B. A. Hay, C. F. Whittington, M. J. Epton, and G. D. Gamble, "FAM13A locus in COPD is independently associated with lung cancer - Evidence of a molecular genetic link between COPD and lung cancer," Application of Clinical Genetics, vol. 4, pp. 1-10, 2011.

[24] B. Wang, H. Zhou, J. Yang et al., "Association of HHIP polymorphisms with COPD and COPD-related phenotypes in a Chinese Han population," Gene, vol. 531, no. 1, pp. 101-105, 2013.

[25] B. Wang, B. Liang, J. Yang et al., "Association of FAM13A polymorphisms with COPD and COPD-related phenotypes in Han Chinese," Clinical Biochemistry, vol. 46, no. 16-17, pp. 1683$1688,2013$.

[26] L. Yang, F. Qiu, X. Lu et al., "Functional Polymorphisms of CHRNA3 Predict Risks of Chronic Obstructive Pulmonary Disease and Lung Cancer in Chinese," PLoS ONE, vol. 7, no. 10, Article ID e46071, 2012.

[27] W. Chen, J. M. Brehm, A. Manichaikul et al., "A genome-wide association study of chronic obstructive pulmonary disease in Hispanics," Annals of the American Thoracic Society, vol. 12, no. 3, pp. 340-348, 2015.

[28] W. J. Kim, Y.-M. Oh, J. H. Lee et al., "Genetic variants in HHIP are associated with FEV1 in subjects with chronic obstructive pulmonary disease," Respirology, vol. 18, no. 8, pp. 1202-1209, 2013.

[29] P.-T. Chuang and A. P. McMahon, "Vertebrate hedgehog signalling modulated by induction of a hedgehog-binding protein," Nature, vol. 397, no. 6720, pp. 617-621, 1999.

[30] Y. Katoh and M. Katoh, "Comparative genomics on HHIP family orthologs," International Journal of Molecular Medicine, vol. 17, no. 2, pp. 391-395, 2006. 
[31] M.-H. Chen, C. W. Wilson, and P.-T. Chuang, "SnapShot: Hedgehog Signaling Pathway," Cell, vol. 130, no. 2, pp. 386-e2, 2007.

[32] M. Van Tuyl and M. Post, "From fruitflies to mammals: Mechanisms of signalling via the Sonic hedgehog pathway in lung development," Respiratory Research, vol. 1, no. 1, pp. 30-35, 2000.

[33] D. Warburton, S. Bellusci, S. De Langhe et al., "Molecular mechanisms of early lung specification and branching morphogenesis," Pediatric Research, vol. 57, no. 5, 2005.

[34] L. L. Rubin and F. J. de Sauvage, "Targeting the Hedgehog pathway in cancer," Nature Reviews Drug Discovery, vol. 5, no. 12, pp. 1026-1033, 2006.

[35] D. N. Watkins, D. M. Berman, S. G. Burkholder, B. Wang, P. A. Beachy, and S. B. Baylin, "Hedgehog signalling within airway epithelial progenitors and in small-cell lung cancer," Nature, vol. 422, no. 6929, pp. 313-317, 2003.

[36] X. Zhou, R. M. Baron, M. Hardin et al., "Identification of a chronic obstructive pulmonary disease genetic determinant that regulates HHIP," Human Molecular Genetics, vol. 21, no. 6, Article ID ddr569, pp. 1325-1335, 2012.

[37] M. G. Foreman, L. Zhang, J. Murphy et al., "Early-onset chronic obstructive pulmonary disease is associated with female sex, maternal factors, and African American race in the COPDGene study," American Journal of Respiratory and Critical Care Medicine, vol. 184, no. 4, pp. 414-420, 2011.

[38] M. T. Dransfield and W. C. Bailey, "COPD: Racial Disparities in Susceptibility, Treatment, and Outcomes," Clinics in Chest Medicine, vol. 27, no. 3, pp. 463-471, 2006.

[39] C. S. Carlson, T. C. Matise, K. E. North et al., "Generalization and Dilution of Association Results from European GWAS in Populations of Non-European Ancestry: The PAGE Study," PLoS Biology, vol. 11, no. 9, Article ID e1001661, 2013.

[40] J.-J. Kim, H.-I. Lee, T. Park et al., "Identification of 15 loci influencing height in a Korean population," Journal of Human Genetics, vol. 55, no. 1, pp. 27-31, 2010.

[41] A. Johannessen, E. R. Omenaas, P. S. Bakke, and A. Gulsvik, "Implications of reversibility testing on prevalence and risk factors for chronic obstructive pulmonary disease: A community study," Thorax, vol. 60, no. 10, pp. 842-847, 2005.

[42] D. B. Hancock, M. Eijgelsheim, J. B. Wilk et al., "Metaanalyses of genome-wide association studies identify multiple loci associated with pulmonary function," Nature Genetics, vol. 42, no. 1, pp. 45-52, 2010.

[43] S. G. Pillai, X. Kong, L. D. Edwards et al., "Loci identified by genome-wide association studies influence different diseaserelated phenotypes in chronic obstructive pulmonary disease," American Journal of Respiratory and Critical Care Medicine, vol. 182, no. 12, pp. 1498-1505, 2010.

[44] J. B. Wilk, L. Djousse, D. K. Arnett et al., "Evidence for major genes influencing pulmonary function in the NHLBI Family Heart Study," Genetic Epidemiology, vol. 19, no. 1, pp. 81-94, 2000 . 

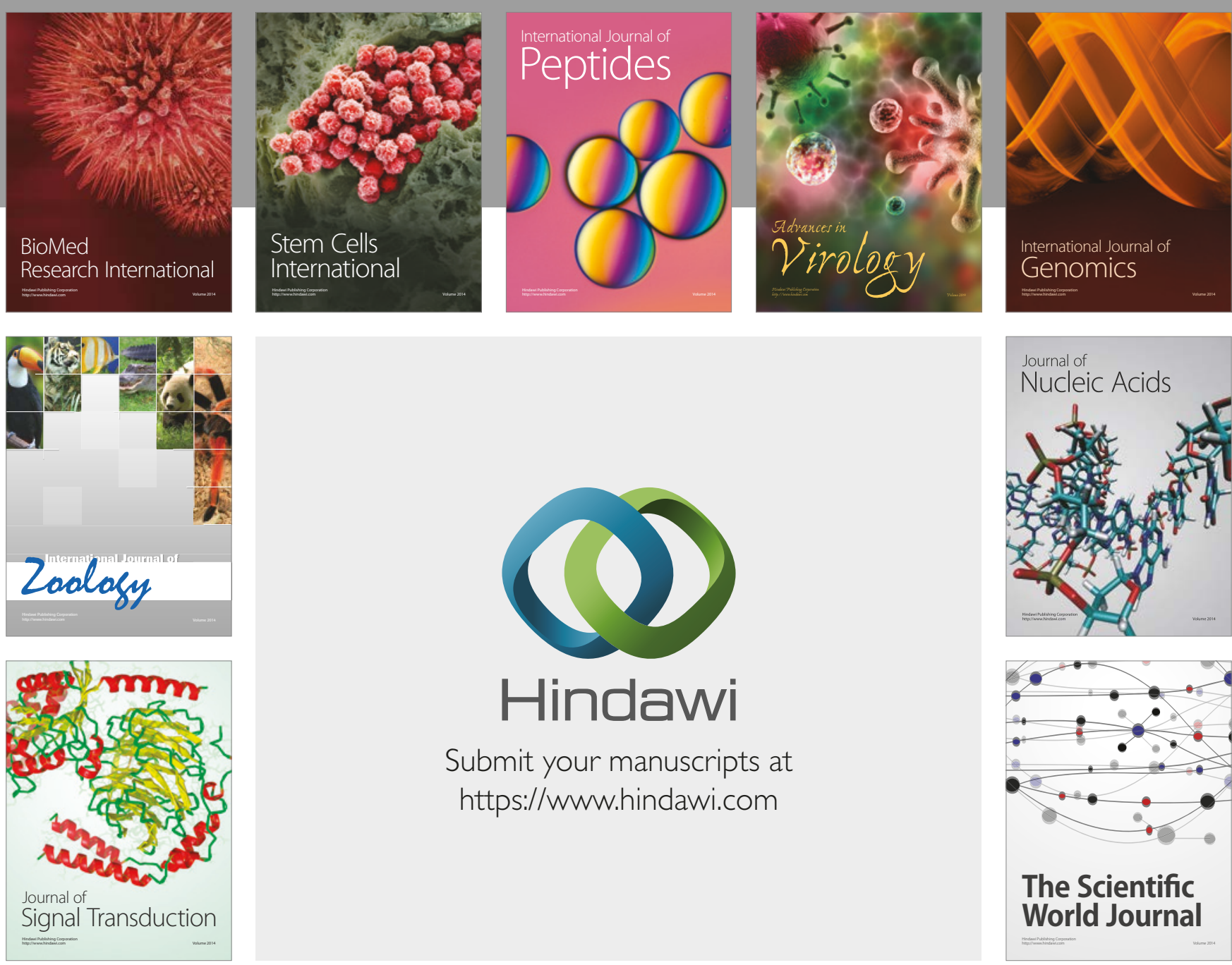

Submit your manuscripts at

https://www.hindawi.com
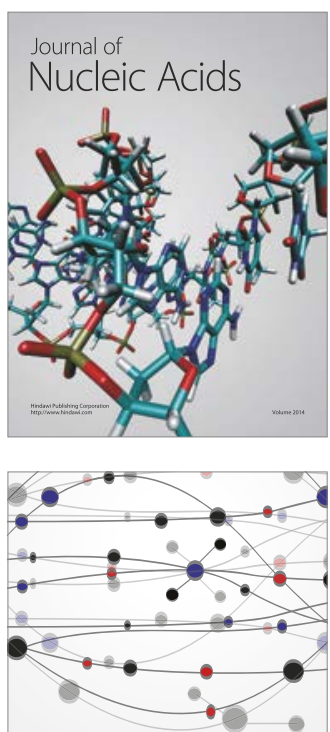

The Scientific World Journal

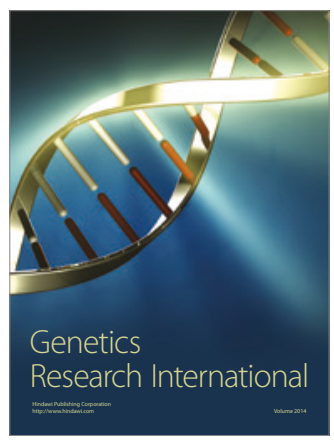

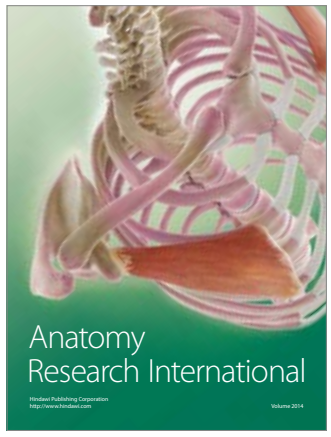

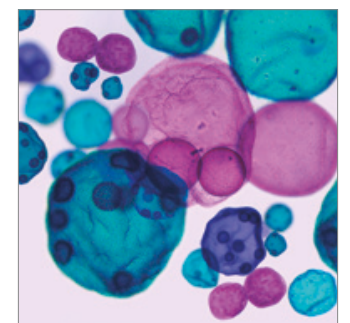

International Journal of Microbiology
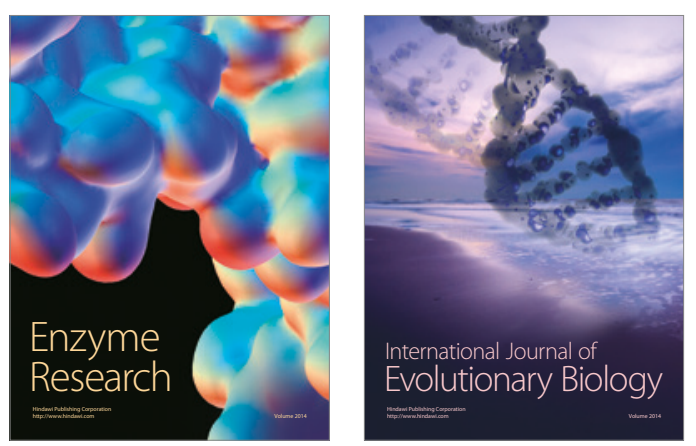
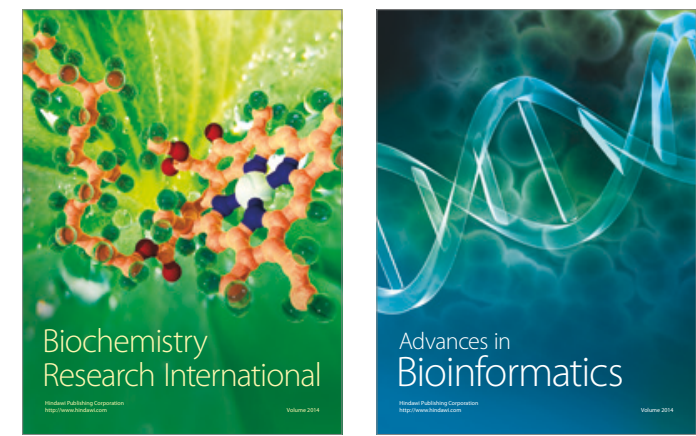

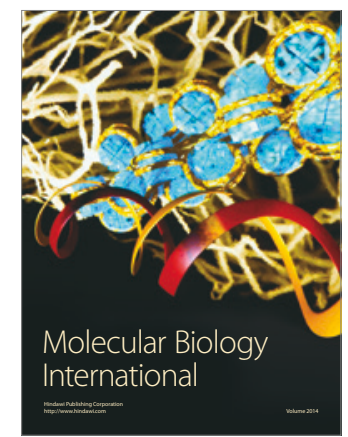

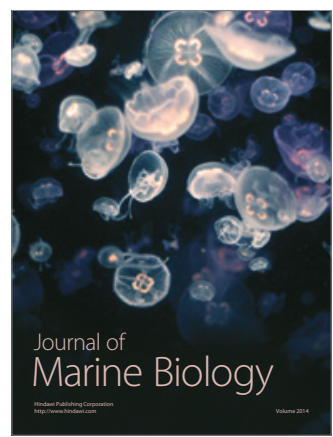

